\title{
The Cauchy problem of the Kadomtsev-Petviashvili hierarchy with arbitrary coefficient algebra
}

Anahita Eslami Rad, Jean-Pierre Magnot, Enrique G. Reyes

To cite this article: Anahita Eslami Rad, Jean-Pierre Magnot, Enrique G. Reyes (2017) The Cauchy problem of the Kadomtsev-Petviashvili hierarchy with arbitrary coefficient algebra, Journal of Nonlinear Mathematical Physics 24: Supplement 1, 103-120, DOI: https://doi.org/10.1080/14029251.2017.1418057

To link to this article: https://doi.org/10.1080/14029251.2017.1418057

Published online: 04 January 2021 


\title{
The Cauchy problem of the Kadomtsev-Petviashvili hierarchy with arbitrary coefficient algebra
}

\author{
Anahita Eslami Rad \\ Departamento de Matemática y Ciencia de la Computación, \\ Universidad de Santiago de Chile, Casilla 307 Correo 2, Santiago, Chile \\ anahita.eslami@usach.cl \\ Jean-Pierre Magnot
}

LAREMA, Université d'Angers, 2 Bd Lavoisier, 49045 Angers cedex 1, France

Lycée Jeanne d'Arc, Avenue de Grande Bretagne, 63000 Clermont-Ferrand, France

jean-pierr.magnot@ac-clermont.fr

Enrique G. Reyes

Departamento de Matemática y Ciencia de la Computación, Universidad de Santiago de Chile, Casilla 307 Correo 2, Santiago, Chile

enrique.reyes@usach.cl,e_g_reyes@yahoo.ca

Received 18 September 2017

Accepted 13 November 2017

\begin{abstract}
Mulase solved the Cauchy problem of the Kadomtsev-Petviashvili (KP) hierarchy in an algebraic category in "Solvability of the super KP equation and a generalization of the Birkhoff decomposition" (Inventiones Mathematicae, 1988), making use of a delicate factorization of an infinite-dimensional group of formal pseudodifferential operators of infinite order. We prove Mulase's factorization theorem in a smooth category in the setting of formal pseudo-differential operators with coefficients in a (non-commutative) algebra equipped with a valuation. As an application, we solve the initial value problem for the KP hierarchy using $r$-matrix theory.
\end{abstract}

\section{Introduction}

Let $L=\partial+a_{1} \partial^{-1}+a_{2} \partial^{-2}+\cdots$ be a (formal) pseudo-differential operator. The KadomtsevPetviashvili (KP) hierarchy is the system

$$
\frac{\partial}{\partial t_{m}} L=\left[\left(L^{m}\right)_{+}, L\right], \quad m \geq 1
$$

in which $\left(L^{m}\right)_{+}$denotes the projection of $L^{m}$ into the space of differential operators. It encodes an infinite number of equations for the coefficients of $L$ for each $m$ and, very importantly, it implies that if $L$ solves the " $t_{m}$ " KP system, so does $L+\varepsilon \frac{\partial}{\partial t_{n}} L$ for any $n$ to first order in $\varepsilon$. Moreover, the KP hierarchy generates symmetries of any equation of the form

$$
\frac{\partial}{\partial t_{m}}\left(L^{n}\right)_{+}-\frac{\partial}{\partial t_{n}}\left(L^{m}\right)_{+}=\left[\left(L^{m}\right)_{+},\left(L^{n}\right)_{+}\right]
$$


a finite system of partial differential equations in three independent variables which becomes (for $\left.n=3, m=2, t_{2}=y, t_{3}=t, 2 a_{1}=u\right)$ the standard Kadomtsev-Petviashvili equation

$$
3 u_{y y}=\partial\left(4 u_{t}-\partial^{3}(u)-6 u \partial(u)\right) .
$$

These remarks appear in [8, Chp. 4]. They imply that integrating the KP hierarchy corresponds to finding the flows of an infinite number of infinitesimal symmetries. In this paper we find these flows: we solve the Cauchy problem for $K P$ in a (smooth) category by means of a generalized version of Mulase's factorization theorem, a factorization of infinite-dimensional Lie groups of formal pseudodifferential operators of infinite order which generalizes the Birkhoff factorization of loop groups, see $[14,15]$ and [17].

We have provided versions of Mulase's theorem in $[12,13]$, and we have considered the Cauchy problem for KP in $[9,13]$. In $[9,12,13]$ we assumed that the coefficients of our formal pseudodifferential operators belong to a commutative algebra of series in infinitely many variables, but in fact, neither commutativity (as in $[9,12,13]$ ) nor series (as in $[14,15]$ ) are needed: as we show here, it is enough to consider formal pseudo-differential operators with coefficients in an arbitrary algebra equipped with a valuation ${ }^{\mathrm{a}}$. We remark that we find interesting to insist on the study of KP in general contexts due to the appearance of Frobenius algebra-valued integrable systems, see [1,22,27], the existence of applications of Mulase's ideas to mathematical physics, see for instance [19, 23, 24], and the possibility of using it as a guide for the study of differential equations in infinite dimensional spaces in the spirit of [5].

With respect to smoothness: in $[12,13]$ we worked mainly in the category of Frölicher spaces. Instead, in the present paper we use diffeologies, a choice imposed on us once we fix our algebraic setting, as we explain briefly in Sections 3 and 4. This context allows us to show existence, uniqueness and smoothness with respect to initial condition of solutions to (1.1).

Definition 1.1. Let $X$ be a set.

- A p-plot of dimension $p$ on $X$ is a map from an open subset $O$ of $\mathbb{R}^{p}$ to $X$.

- A diffeology on $X$ is a set $\mathscr{P}$ of plots on $X$ such that, for all $p \in \mathbb{N}$,

- All constant maps $\mathbb{R}^{p} \rightarrow X$ are in $\mathscr{P}$;

- Let $\left\{f_{i}: O_{i} \rightarrow X\right\}_{i \in I}$ be a family of compatible maps that extend to a map $f: \bigcup_{i \in I} O_{i} \rightarrow X$. If $\left\{f_{i}: O_{i} \rightarrow X\right\}_{i \in I} \subset \mathscr{P}$, then $f \in \mathscr{P}$.

- Let $f: O \subset \mathbb{R}^{p} \rightarrow X$ be in $\mathscr{P}$. If $q \in \mathbb{N}, O^{\prime}$ is an open subset of $\mathbb{R}^{q}$, and $g$ a smooth map (in the usual sense) from $O^{\prime}$ to $O$, then $f \circ g \in \mathscr{P}$.

If $\mathscr{P}$ is a diffeology on $X$, then $(X, \mathscr{P})$ is called a diffeological space and, if $\left(X^{\prime}, \mathscr{P}^{\prime}\right)$ is another diffeological space, we say that a map $f: X \rightarrow X^{\prime}$ is smooth if and only if $f \circ \mathscr{P} \subset \mathscr{P}^{\prime}$.

This general approach to smoothness was introduced in Mathematical Physics by J.-M. Souriau, see [21] and the recent treatise [10], and in Algebraic Topology (using convex subsets of $\mathbb{R}^{p}$ instead of open sets as in Definition 1.1) by K.T. Chen, see [4] and also [25]. If $X$ is a manifold, then $X$ is a diffeological space with $p$-plots being the $C^{\infty}$ maps from $O \subseteq \mathbb{R}^{p}$ to $X$, but the fact that diffeologies

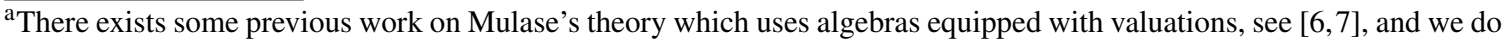
acknowledge partial inspiration from these papers. However, it appears to us that they must be read quite critically. For instance, we find that completeness of the valuation is needed at some crucial points (e.g. Lemma 2), and this assumption is not stated in $[6,7]$. Also, the construction of groups of formal pseudo-differential operators appearing therein needs to be refined, see [15] and Definition 5 below.
} 
allow us to consider smooth maps without resorting to charts means that this concept is of really wide applicability. Several applications are considered in [10]; we also refer to [12, 13, 26] and [2].

\section{Preliminaries}

\subsection{The algebra of formal pseudo-differential operators}

Let $A$ be an associative $K$-algebra with unit 1, in which $K$ is a field of characteristic zero. Inspired by Chen [4], we say that $A$ is a diffeological topological algebra if:

- The field $K$ is equipped with discrete topology and with discrete diffeology (see [10, p. 10]). Then, addition, multiplication and inversion on $K^{*}=K \backslash\{0\}$ are continuous and smooth.

- The ring $A$ is a topological ring equipped with a diffeology $\mathscr{P}$ such that addition, multiplication, and inversion on $A^{*}$ (= set of units of $A$ ) are smooth and the plots of $\mathscr{P}$ are continuous.

- The scalar multiplication $K \times A \rightarrow A$ is continuous and smooth if we equip $K \times A$ with the product diffeology (see [10, p. 32]).

Here and henceforth, by smooth we mean smooth in the sense of Definition 1.1.

We assume that $A$ is equipped with a derivation, that is, with a $K$-linear map $D: A \rightarrow A$ such that $D(f \cdot g)=(D f) \cdot g+f \cdot(D g)$ for all $f, g \in A$.

Let $\xi$ be a formal variable. The algebra of symbols over $A$ is the vector space

$$
\Psi_{\xi}(A)=\left\{P_{\xi}=\sum_{v \in \mathbf{Z}} a_{v} \xi^{v}: a_{v} \in A, a_{v}=0 \text { for } v \gg 0\right\},
$$

equipped with the associative multiplication $\circ$ given by

$$
P_{\xi} \circ Q_{\xi}=\sum_{k \geq 0} \frac{1}{k !} \frac{\partial^{k} P_{\xi}}{\partial \xi^{k}} D^{k} Q_{\xi}
$$

with the prescription that multiplication on the right hand side of (2.1) is standard multiplication of Laurent series in $\xi$ with coefficients in $A$, see [11]. Equation (2.1) is equivalent to the following generalized Leibniz rule

$$
\left(\sum_{\mu \in \mathbb{Z}} a_{\mu} \xi^{\mu}\right) \circ\left(\sum_{\kappa \in \mathbb{Z}} b_{\kappa} \xi^{\kappa}\right)=\sum_{v \in \mathbb{Z}}\left(\sum_{\mu \in \mathbb{Z}} \sum_{k=0}^{\infty}\left(\begin{array}{c}
\mu \\
k
\end{array}\right) a_{\mu} b_{v-\mu+k}^{(k)}\right) \xi^{v}
$$

in which $b_{\mu}^{(k)}=D^{k}\left(b_{\mu}\right)$ and the combinatorial symbol is extended to arbitrary values of $\alpha \in \mathbb{Z}$ via

$$
\left(\begin{array}{l}
\alpha \\
k
\end{array}\right)=\left\{\begin{array}{cc}
0 & k>\alpha \geq 0 \\
(-1)^{k}\left(\begin{array}{c}
-\alpha+k-1 \\
k
\end{array}\right) & \alpha<0
\end{array}\right.
$$

The algebra of formal pseudo-differential operators over $A$ is the vector space

$$
\Psi(A)=\left\{P=\sum_{v \in \mathbb{Z}} a_{v} D^{v}: a_{v} \in A, a_{v}=0 \text { for } v \gg 0\right\}
$$


equipped with the unique multiplication which makes the map $\sum_{v \in \mathbb{Z}} a_{v} \xi^{v} \mapsto \sum_{v \in \mathbb{Z}} a_{v} D^{v}$ an algebra homomorphism. The algebra $\Psi(A)$ is associative but not commutative, and so it possesses a natural non-trivial Lie algebra structure.

If $A$ is a diffeological topological algebra and $D$ is continuous and smooth, then operations on the coefficients of formal pseudo-differential operators are continuous and smooth, coefficient by coefficient, see [12,13]. Moreover, $\Psi(A)$ is a diffeological $K$-algebra for the restriction of the diffeology of $A^{\mathbb{Z}}$ (the space of $\mathbb{Z}$-sequences of $A$ ) to $\Psi(A)$, see [10, pp. 18, 34], and the function $[]:, \Psi(A) \times \Psi(A) \rightarrow \Psi(A)$ is smooth.

The Lie algebra $\Psi(A)$ admits the decomposition $\Psi(A)=\mathscr{I}_{A} \oplus \mathscr{D}_{A}$, in which $\mathscr{D}_{A}$ is the Lie subalgebra of all differential operators of order greater or equal to zero, and $\mathscr{I}_{A}$ is the Lie subalgebra of all formal pseudo-differential operators of order at most -1 . This observation is crucial for our analysis of the initial value problem of the KP hierarchy to be carried out in Section 4.

\subsection{Algebras with (diffeological) valuations}

We fix an algebra $A$ as in Section 2.1 and we assume that it is equipped with a non-trivial valuation (Definition 2.1 below). Our main references on valuations are [3,20].

Definition 2.1. A valuation on an algebra $A$ is a map $\sigma: A \rightarrow \mathbb{Z} \cup\{\infty\}$ which satisfies the following properties for all $a, b$ in $A$ and $k \in K$ :

- $\sigma(a)=\infty$ if and only if $a=0$, and $\sigma(1)=0$

- $\sigma(a b)=\sigma(a)+\sigma(b)$, and $\sigma(k a)=\sigma(a)$

- $\sigma(a+b) \geq \min (\sigma(a), \sigma(b))$.

The following conventions are implicit in the definition above: for all $n \in \mathbb{Z}$ we set $n+\infty=\infty$; $\infty+\infty=\infty ; n<\infty$. We also note three properties of a valuation $\sigma: \sigma(-1)=0 ; \sigma(-x)=\sigma(x)$ for all $x \in A ; \sigma(a+b)=\min (\sigma(a), \sigma(b))$ whenever $\sigma(a) \neq \sigma(b)$.

A valuation allows us to equip $A$ with a topology. For $\alpha \in \mathbb{Z}$ and $x_{0} \in A$ we set

$$
V_{\alpha}\left(x_{0}\right)=\left\{x \in A: \sigma\left(x-x_{0}\right)>\alpha\right\}
$$

we easily see that for each $x_{0} \in A$, the collection $\left\{V_{\alpha}\left(x_{0}\right)\right\}_{\alpha \in \mathbb{Z}}$ is a basis of neighbourhoods for a first countable Hausdorff topology on $A$. This topology is metrizable: we define the absolute value $|x|=c^{\sigma(x)}$ for $x \in A$ and a fixed real number $0<c<1$. Then, the following holds:

- $|x| \geq 0,|x|=|k x|$ for $k \in K^{*}$, and $|x|=0$ if and only if $x=0$;

- $|x y|=|x||y|$;

- $|x+y| \leq \max \{|x|,|y|\}$, and $|x+y|=\max \{|x|,|y|\}$ if $|x| \neq|y|$.

If we set $d(x, y)=|x-y|$ we obtain a metric $d$ on $A$ and the metric topology coincides with the topology introduced above. In particular, the sets $V_{\alpha}\left(x_{0}\right)$ coincide with the balls $\left\{x \in A:|x|<c^{\alpha}\right\}$ and they are both open and closed, see [20, Prop. 18.4].

Hereafter we assume that the derivation $D$ is compatible with the valuation $\sigma$ in the sense that $\sigma(D(x)) \geq \sigma(x)$ for all $x \in A$ or, equivalently, that $|D(a)| \leq|a|$ for all $a \in A$. In the topology just defined, $A$ becomes a topological algebra and $D$ a continuous derivation if we equip the field $K$ with the discrete topology.

Remark 2.1. In [15] Mulase used as $A$ an algebra of formal power series in an infinite number of variables $\tau_{1}, \tau_{2}, \cdots$, equipped with the valuation $v_{a l}$ determined by the rule $v_{a l}\left(\tau_{k}\right)=k$. This case 
is reviewed in [9], see also [12,13]. Formal power series in infinitely many variables are introduced in [3, Section 1.5.5].

Lemma 2.1. (see [20, Chp. 2]) Let $\left\{a_{n}\right\}_{n \geq 0}$ be a sequence in A. Then,

(1) If $\lim _{n \rightarrow \infty} a_{n}=a \in A$ and $a \neq 0$, then $\left|a_{n}\right|=|a|$ for large $n$. Thus, convergent sequences are bounded.

(2) The sequence $\left\{a_{n}\right\}_{n \geq 0}$ is a Cauchy sequence if and only if $\lim _{n \rightarrow \infty}\left|a_{n+1}-a_{n}\right|=0$.

(3) The series $\sum_{n=0}^{\infty} a_{n}$ converges if and only if $\lim _{n \rightarrow \infty} a_{n}=0$. Moreover, if $\lim _{n \rightarrow \infty} a_{n}=0$, then $\left|\sum_{n=0}^{\infty} a_{n}\right|<\max _{n \geq 0}\left|a_{n}\right|$.

(4) Assume that $\lim _{n \rightarrow \infty} a_{m n}=0$ for each $m$, and that $\lim _{m \rightarrow \infty} a_{m n}=0$ uniformly in $n$. Then, the double series $\sum_{(m, n)} a_{m n}$ exists and

$$
\sum_{(m, n)} a_{m n}=\sum_{m=0}^{\infty} \sum_{n=0}^{\infty} a_{m n}=\sum_{n=0}^{\infty} \sum_{m=0}^{\infty} a_{m n}=\lim _{N \rightarrow \infty} \sum_{m=0}^{N} \sum_{n=0}^{N} a_{m n} .
$$

Now we let $\hat{A}$ be the completion of the metric space $(A, d)$, and we extend $D$ to a continuous derivation on $\hat{A}$ which we also call $D$. We keep denoting the extension of the absolute value from $A$ to $\hat{A}$ by $|\cdot|$. We note that the algebra $A$ considered by Mulase in [15] (see Remark 2.1) is complete, as explained in [3, Section 1.5.5].

We define a valuation $\hat{\sigma}: \hat{A} \rightarrow \mathbb{R} \cup\{\infty\}$ as $\hat{\sigma}(a)=\log _{c}(|a|)$. Clearly, $\hat{\sigma}(x)=\sigma(x)$ for $x \in A \subseteq \hat{A}$. Moreover, the range of $\hat{\sigma}$ is exactly the range of $\sigma$, see [20, Exercise 8.D]. This fact implies that $\hat{\sigma}$ is continuous in the topology of $\hat{A}$ induced by $|\cdot|$.

There exist two standard structures associated to the valuation $\hat{\sigma}$ on $\hat{A}$ which we will use in the following sections. The closed subring $\mathscr{O}_{\hat{A}}=\{a \in \hat{A}: \hat{\sigma}(a) \geq 0\}$, and the two-sided ideal $\mathscr{P}_{\hat{A}}=\left\{a \in \mathscr{O}_{\hat{A}}: \hat{\sigma}(a)>0\right\}$. If $A$ is a diffeological topological algebra, $\mathscr{O}_{\hat{A}}$ and $\mathscr{P}_{\hat{A}}$ carry natural underlying diffeologies. Since it is easy to check that the derivation $D$ on $\hat{A}$ is compatible with $\hat{\sigma}$, we have $D\left(\mathscr{P}_{\hat{A}}\right) \subset \mathscr{P}_{\hat{A}}$; it follows that $D$ is well-defined on the quotient ring $\mathscr{O}_{\hat{A}} / \mathscr{P}_{\hat{A}}$. We let $\pi: \mathscr{O}_{\hat{A}} \rightarrow \mathscr{O}_{\hat{A}} / \mathscr{P}_{\hat{A}}$ be the canonical projection. If $A$ is a diffeological topological algebra, the map $\pi$ is continuous and smooth.

Now we need to specify the diffeologies carried by the topological algebras $A$ and $\hat{A}$ so that they become diffeological topological algebras. For all $p \in \mathbb{Z}$, the quotient vector space projection $\pi_{p}: A \rightarrow A / A_{p}$, in which $A_{p}=\{a \in A: \sigma(a) \geq p\}$, extends to $\hat{A}$ in the following way: for $\hat{a} \in \hat{A}$, we set $\pi_{p}(\hat{a})=a+A_{p}$ if and only if $\sigma(a)=\hat{\sigma}(\hat{a})$ and $\hat{\sigma}(a-\hat{a}) \geq p$. It is possible to find such an $a \in A$ because there exists $\left(a_{n}\right) \in A^{\mathbb{N}}$ such that $\lim a_{n}=\hat{a}$, and Lemma 2.1 implies that $\sigma\left(a_{n}\right)=\hat{\sigma}(\hat{a})$ for large enough $n$. That $\pi_{p}$ is well defined follows from the strong triangle inequality appearing in Definition 2.1.

We note that due to the presence of quotients, the use of diffeologies is more natural than the use of Frölicher spaces as in [12,13]. In fact, in contradistinction with diffeologies (see [10, p. 27] for the construction of the quotient diffeology) the latter structure does not pass easily to quotients, as explained in [10], see also [26].

Definition 2.2. We equip the quotients $A / A_{p}$ with their quotient diffeology. The completion $\hat{A}$ is equipped with the pull-back diffelogy with respect to the family of maps $\left\{\pi_{p} ; p \in \mathbb{Z}\right\}$ (see [10, p. 32]). The valuation $\sigma$ of $A$ is called a diffeological valuation if and only if the diffeology of $A$ is the pull-back of the diffeology of $\hat{A}$ and all plots are continuous in the valuation topologies of $A$ and $\hat{A}$. 
Assumption (A): Hereafter we assume that the topological algebras $A$ and $\hat{A}$ are equipped with diffeological valuations, and that $D$ is smooth with respect to the diffeology on $\hat{A}$, which implies that its restriction to $A$ is also smooth.

Theorem 2.1. Let $x \in O \subset \mathbb{R}^{p} \mapsto\left\{a_{n}(x)\right\}_{n \geq 0} \in A^{\mathbb{Z}}$ be a smooth plot on $A^{\mathbb{Z}}$ for the product diffeology such that $\inf _{x \in O, n \in \mathbb{N}} \sigma\left(a_{n}(x)\right)>-\infty$. We assume that for each $x \in O$ the sequence $\left\{a_{n}(x)\right\}_{n \geq 0}$ converges to $\hat{a}(x) \in \hat{A}$, that $\lim _{n \rightarrow \infty} \inf _{x \in O} \hat{\sigma}\left(a_{n}(x)-\hat{a}(x)\right)=+\infty$, and that (A) holds. Then, the map $x \in O \mapsto \hat{a}(x)$ is smooth, that is, "lim" is a smooth map.

Proof. (Sketch) Let $\pi_{p}$ be projection on $A / A_{p+1}$. By our "uniform convergence" assumption,

$$
\forall p \in \mathbb{Z}, \exists N \in \mathbb{N}, \forall n>N, \hat{\sigma}\left(a_{n}(x)-\hat{a}(x)\right)>p .
$$

Thus, the well-defined projection map $\prod_{p \in \mathbb{Z}} \pi_{p}: \hat{A} \rightarrow \prod_{p \in \mathbb{Z}} A / A_{p}$ defined before is smooth. It follows from the definition of the pull-back diffeology that the limit is smooth.

The diffeology on $\hat{A}$ is spanned by plots that verify the conditions of Theorem 2.1. Thus, all smoothness properties have to be checked only for these generating plots. We use this observation to prove the following:

Proposition 2.1. Multiplication is a smooth operation on the algebra $\hat{A}$.

Proof. We fix two generating plots $(\hat{a}(x), \hat{b}(x)) \in\left(C^{\infty}(O, \hat{A})\right)^{2}$, and we let $\left(a_{n}(x)\right)$ and $\left(b_{n}(x)\right)$ be two corresponding sequences of plots in $A$ converging to $\hat{a}(x)$ and $\hat{b}(x)$ respectively, so that

$$
\lim _{n \rightarrow \infty} \inf _{x \in O} \hat{\sigma}\left(a_{n}(x)-\hat{a}(x)\right)=\lim _{n \rightarrow \infty} \inf _{x \in O} \hat{\sigma}\left(b_{n}(x)-\hat{b}(x)\right)=+\infty .
$$

Moreover we have that for all $p \in \mathbb{Z}$ there exist $N(p), N^{\prime}(p) \in \mathbb{N}$ such that for all $x \in O$

$$
\pi_{p} \circ \hat{a}(x)=\pi_{p} \circ a_{n}(x) \forall n>N(p) \quad \text { and } \quad \pi_{p} \circ \hat{a}(x)=\pi_{p} \circ b_{n}(x) \forall n>N^{\prime}(p) .
$$

We can assume with no loss of generality that $\forall n, \sigma\left(a_{n}(x)\right)=\hat{\sigma}(\hat{a}(x))$ and $\sigma\left(b_{n}(x)\right)=\hat{\sigma}(\hat{b}(x))$. We observe that

$$
\begin{aligned}
\lim _{n \rightarrow \infty} \inf _{x \in O} \sigma\left(\hat{a} \hat{b}-a_{n} b_{n}\right)(x) & =\liminf _{n \rightarrow \infty} \sigma\left(\hat{a}\left(\hat{b}-b_{n}\right)+\left(\hat{a}-a_{n}\right) b_{n}\right)(x) \\
& \geq \max \left\{\hat{\sigma}(\hat{a}) \lim _{n \rightarrow \infty} \inf _{x \in O} \hat{\sigma}\left(\hat{b}-b_{n}\right), \liminf _{n \rightarrow \infty} \hat{x} \hat{\sigma}\left(\hat{a}-a_{n}\right)+\sigma\left(b_{n}\right)\right\}(x) \\
& =+\infty .
\end{aligned}
$$

It follows that the projections of $\hat{a}(x) \hat{b}(x)$ on $A / A_{p}$ are defined by the projections of $a_{n}(x) b_{n}(x)$ for $n$ large enough, which are constant in $n$ and smooth in $x$. In other words, for all $p \in \mathbb{Z}$ there exists $N^{\prime \prime}$ such that for all $n>N^{\prime \prime}$ we have $\pi_{p}(\hat{a}(x) \hat{b}(x))=\pi_{p}\left(a_{n}(x) b_{n}(x)\right)$. We conclude that the map $x \mapsto \hat{a}(x) \hat{b}(x)$ is a plot of the diffeology of $\hat{A}$, which shows that multiplication is smooth.

Remark 2.2. We stress that the diffeology of the completion of $A$ is deduced from the diffeology of $A$ via the quotients $A / A_{p}$, and that the application lim is automatically smooth. These facts seem to make essential use of our non-archimedean valuation setting. For example, when $\mathbb{Q}$ is completed to $\mathbb{R}$ for the classical absolute value, such a phenomenon does not appear: $\mathbb{Q}$ has only discrete diffeology, whereas the diffeology of $\mathbb{R}$ is much richer and it cannot be obtained from the (discrete) diffeology of $\mathbb{Q}^{\mathbb{N}}$. 


\section{Pseudo-differential operators and Mulase's theorem}

\subsection{Formal Lie groups and the Mulase decomposition}

Definition 3.1. The spaces of formal pseudo-differential and differential operators of infinite order are, respectively,

$$
\begin{array}{r}
\widehat{\Psi}(\hat{A})=\left\{P=\sum_{\alpha \in \mathbb{Z}} a_{\alpha} D^{\alpha}: a_{\alpha} \in \hat{A} \text { and } \exists A_{P}, B_{P} \in \mathbb{R}^{+} \text {and } M_{P}, N_{P}, L_{P} \in \mathbb{Z}^{+}\right. \text {so that } \\
\left.M_{P} \geq N_{P},\left|a_{\alpha}\right|<\frac{A_{P}}{\alpha-N_{P}} \forall \alpha>M_{P} \text {, and }\left|a_{\alpha}\right|<B_{P} \forall \alpha<-L_{P}\right\}
\end{array}
$$

and

$$
\widehat{\mathscr{D}}_{\hat{A}}=\left\{P=\sum_{\alpha \in \mathbb{Z}} a_{\alpha} D^{\alpha}: P \in \widehat{\Psi}(\hat{A}) \text { and } a_{\alpha}=0 \text { for } \alpha<0\right\} .
$$

We note that the use of the completion $\widehat{A}$ instead of $A$ in Definition 3.1 is crucial in order to equip $\widehat{\Psi}(\hat{A})$ with an algebra structure, see Lemma 3.1 below. We also note that the growth conditions in (3.1) are needed in the construction of our group of formal pseudo-differential operators of infinite order and of an exponential map, see Definition 3.2, Proposition 3.1 and Lemma 3.3. Finally, we remark that if $P=\sum_{\alpha \in \mathbb{Z}} a_{\alpha} D^{\alpha} \in \widehat{\Psi}(\hat{A})$, then $\lim _{\alpha \rightarrow \infty} a_{\alpha}=0$, and also that there exists $C_{P}>0$ such that $\left|a_{\alpha}\right|<C_{P}$ for all $\alpha \in \mathbb{Z}$. Moreover, the definition of $|\cdot|$ implies that $\hat{A}$ is contained in $\widehat{\Psi}(\hat{A})$ and, under our assumption $(\mathbf{A}), \widehat{\Psi}(\hat{A})$ and $\widehat{\mathscr{D}}(\hat{A})$ are diffeological spaces.

Lemma 3.1. The space $\widehat{\Psi}(\hat{A})$ has an algebra structure and $\widehat{\mathscr{D}}_{\hat{A}}$ is a subalgebra of $\widehat{\Psi}(\hat{A})$. Moreover, under assumption $(\boldsymbol{A}), \widehat{\Psi}(\hat{A})$ and $\widehat{\mathscr{D}}_{\hat{A}}$ are diffeological topological $K$-algebras.

Proof. Let $P=\sum_{\alpha \in \mathbb{Z}} a_{\alpha} D^{\alpha}$ and $Q=\sum_{\mu \in \mathbb{Z}} b_{\mu} D^{\mu}$ be elements of $\widehat{\Psi}(\hat{A})$. We set

$$
P Q=\sum_{v \in \mathbb{Z}}\left[\sum_{\alpha \in \mathbb{Z}} \sum_{k=0}^{\infty}\left(\begin{array}{l}
\alpha \\
k
\end{array}\right) a_{\alpha} b_{v-\alpha+k}^{(k)}\right] D^{v}
$$

We denote the coefficient of $D^{v}$ in $P Q$ by $c_{v}$. The operations that define the map $(a, b) \mapsto c$ are smooth, coefficient by coefficient. We now check that $c_{v}$ exists and that it belongs to $\hat{A}$ for all $v \in \mathbb{Z}$.

$$
c_{v}=\sum_{\alpha \in \mathbb{Z}} \sum_{k=0}^{\infty}\left(\begin{array}{l}
\alpha \\
k
\end{array}\right) a_{\alpha} b_{v-\alpha+k}^{(k)}=\sum_{\alpha=0}^{\infty} \sum_{k=0}^{\alpha}\left(\begin{array}{l}
\alpha \\
k
\end{array}\right) a_{\alpha} b_{v-\alpha+k}^{(k)}+\sum_{\beta=1}^{\infty} \sum_{k=0}^{\infty}\left(\begin{array}{c}
-\beta \\
k
\end{array}\right) a_{-\beta} b_{v+\beta+k}^{(k)}
$$

We call $s_{1}(v)$ and $s_{2}(v)$ the first and second summation respectively; we use the notations introduced in Definition 3.1 and in the remarks made thereafter. We show that $s_{1}$ exists. We set 
$S_{n}=\sum_{\alpha=0}^{n} \sum_{k=0}^{\alpha}\left(\begin{array}{l}\alpha \\ k\end{array}\right) a_{\alpha} b_{v-\alpha+k}^{(k)}$. Then,

$$
\begin{aligned}
\left|S_{n+1}-S_{n}\right| & =\left|\sum_{k=0}^{n+1}\left(\begin{array}{c}
n+1 \\
k
\end{array}\right) a_{n+1} b_{v+k-(n+1)}^{(k)}\right| \\
& \leq \max _{0 \leq k \leq n+1}\left\{\left|\left(\begin{array}{l}
\alpha \\
k
\end{array}\right) a_{n+1} b_{v+k-(n+1)}^{(k)}\right|\right\} \\
& \leq\left|a_{n+1}\right| \max _{0 \leq k \leq n+1}\left\{\left|b_{v+k-(n+1)}\right|\right\} \\
& \leq\left|a_{n+1}\right| C_{Q} .
\end{aligned}
$$

Thus, $\lim _{n \rightarrow \infty}\left|S_{n+1}-S_{n}\right|=0$. It follows that $\left\{S_{n}\right\}$ is a Cauchy sequence in $\hat{A}$, and we conclude that $s_{1}(v)=\lim _{n \rightarrow \infty} S_{n}$.

Now we prove that $s_{2}(v)$ exists using part (4) of Lemma 2.1: we have that $\left|a_{-\beta} b_{v+\beta+k}^{(k)}\right| \leq$ $\left|a_{-\beta}\right|\left|b_{v+\beta+k}\right|$, and the right hand side converges to zero as $k \rightarrow \infty$ for each $\beta$; also, $\left|a_{-\beta} b_{v+\beta+k}^{(k)}\right| \leq$ $B_{P}\left|b_{v+\beta+k}\right|$ for $\beta$ large enough, and the right hand side tends to zero uniformly in $k$ as $\beta \rightarrow \infty$. Thus, we conclude that $s_{2}(v)$ exists, and so $c_{v}$ is a well defined element of $\hat{A}$ for all $v \in \mathbb{Z}$.

Now we show that $c_{v}$ satisfies the growth conditions appearing in Definition 3.1. Since $P, Q$ are in $\widehat{\Psi}(\hat{A})$, there exist numbers $A_{P}, A_{Q}, B_{P}, B_{Q} \in \mathbb{R}^{+}, N_{P}, N_{Q} \in \mathbb{Z}^{+}$and $M_{P}, L_{P}, M_{Q}, L_{Q} \in \mathbb{Z}^{+}$such that

$$
\begin{array}{cll}
\left|a_{\alpha}\right|<\frac{A_{P}}{\alpha-N_{P}} & \text { for } \alpha>M_{P}, & \left|b_{\beta}\right|<\frac{A_{Q}}{\beta-N_{Q}} \quad \text { for } \beta>M_{Q}, \\
\left|a_{\alpha}\right|<B_{P} & \text { for } \alpha<-L_{P}, & \left|b_{\beta}\right|<B_{Q} \quad \text { for } \beta<-L_{Q} .
\end{array}
$$

We estimate $c_{v}$ using (3.3):

$$
\begin{aligned}
\left|c_{v}\right| & =\left|\sum_{\alpha=0}^{\infty} \sum_{k=0}^{\alpha}\left(\begin{array}{l}
\alpha \\
k
\end{array}\right) a_{\alpha} b_{v-\alpha+k}^{(k)}+\sum_{k=0}^{\infty} \sum_{\beta=1}^{\infty}(-1)^{k}\left(\begin{array}{c}
k+\beta-1 \\
k
\end{array}\right) a_{-\beta} b_{v+\beta+k}^{(k)}\right| \\
& =\left|s_{1}(v)+s_{2}(v)\right| \\
& \leq \max \left\{\left|s_{1}(v)\right|,\left|s_{2}(v)\right|\right\},
\end{aligned}
$$

in which $s_{1}(v)$ and $s_{2}(v)$ are defined as before. We set $s_{1}(v)_{\alpha}=\sum_{k=0}^{\alpha}\left(\begin{array}{l}\alpha \\ k\end{array}\right) a_{\alpha} b_{v-\alpha+k}^{(k)}$, so that $s_{1}(v)=\sum_{\alpha=0}^{\infty} s_{1}(v)_{\alpha}$. Since we already know that $s_{1}(v)$ exists, we obtain that $\lim _{\alpha \rightarrow \infty} s_{1}(v)_{\alpha}=0$. Then, Lemma 1 implies that

$$
\left|s_{1}(v)\right| \leq \max _{0 \leq \alpha}\left\{\left|\sum_{k=0}^{\alpha}\left(\begin{array}{l}
\alpha \\
k
\end{array}\right) a_{\alpha} b_{v-\alpha+k}^{(k)}\right|\right\} \leq \max _{0 \leq \alpha} \max _{0 \leq k \leq \alpha}\left|a_{\alpha} b_{v-\alpha+k}\right| .
$$

Now we consider $s_{2}(v)$. Using Lemma 1 again we can write $s_{2}(v)=\lim _{N \rightarrow \infty} d_{N}$ in which

$$
d_{N}=\sum_{k=0}^{N} \sum_{\beta=1}^{N}(-1)^{k}\left(\begin{array}{c}
k+\beta-1 \\
k
\end{array}\right) a_{-\beta} b_{v+\beta+k}^{(k)} .
$$


Let us assume that $s_{2}(v) \neq 0$. Then, there exists $N_{0}$ such that for all $N \geq N_{0}$ we have

$$
\begin{aligned}
\left|s_{2}(v)\right|=\left|d_{N}\right| & \leq \max _{0 \leq k \leq N} \max _{1 \leq \beta \leq N}\left\{\left|a_{\beta}\right|\left|b_{v+\beta+k}\right|\right\} \\
& \leq \max _{1 \leq \beta \leq N}\left|a_{\beta}\right|\left\{\max _{0 \leq k \leq N} \max _{1 \leq \beta \leq N}\left|b_{v+\beta+k}\right|\right\} .
\end{aligned}
$$

We are ready to estimate $\left|s_{1}(v)\right|$ and $\left|s_{2}(v)\right|$ near $\pm \infty$. Set $M=\max \left\{M_{P}, M_{Q}\right\}, L=\max \left\{L_{P}, L_{Q}\right\}$, $A=\max \left\{A_{P}, A_{Q}\right\}, B=\max \left\{B_{P}, B_{Q}\right\}, N=\max \left\{N_{P}, N_{Q}\right\}$.

(1) We use (3.5). We fix $v>2 M$ and we consider two cases, (a) $\alpha \leq M$ and (b) $\alpha>M$.

If ( $a$ ) holds, then $v-\alpha+k>M+k>M$, and so

$$
\left|a_{\alpha} b_{v-\alpha+k}\right| \leq\left|a_{\alpha}\right| \frac{A}{v-\alpha+k-N} \leq\left|a_{\alpha}\right| \frac{A}{v-M+k-N} \leq\left|a_{\alpha}\right| \frac{A}{v-(M+N)} .
$$

If (b) holds, then $\left|a_{\alpha}\right|<\frac{A}{\alpha-N}$ and therefore

$$
\left|a_{\alpha}\right|\left|b_{v-\alpha+k}\right|<\frac{A}{\alpha-N}\left|b_{v-\alpha+k}\right| .
$$

We have two sub-cases depending on $v-\alpha$ :

If $v-\alpha>M$, then $v-\alpha+k>M+k$ and therefore

$$
\left|a_{\alpha}\right|\left|b_{v-\alpha+k}\right|<\frac{A}{\alpha-N} \frac{A}{v-\alpha+k-N}<\frac{A}{\alpha-N} \frac{A}{v-M+k-N} \leq \frac{A}{\alpha-N} \frac{A}{v-(M+N)} .
$$

If $v-\alpha \leq M$, then (3.7) implies $\left|a_{\alpha}\right|\left|b_{v-\alpha+k}\right|<\frac{A}{\alpha-N} C_{Q}$. But $v-M \leq \alpha$, so that $\left|a_{\alpha}\right|\left|b_{v-\alpha+k}\right|<\frac{A}{v-M-N} C_{Q}$. We conclude that there exists $B \in \mathbb{R}$ such that

$$
s_{1}(v)<\frac{B}{v-(M+N)} .
$$

On the other hand, (3.5) implies that we can assure that $v-\alpha+k<-L$ for $v<0$ and $|v|$ large enough, and so there exists $\tilde{B}>0$ such that for $v$ near $-\infty$ we have

$$
\left|s_{1}(v)\right| \leq \tilde{B} .
$$

(2) We use (3.6). We choose $v>M$ so that $v+\beta+k>M$. Then, there exists $E>0$ such that

$$
\left|s_{2}(v)\right| \leq E \frac{A}{v+\beta+k-N} \leq \frac{E A}{v-N} .
$$

On the other hand, (3.6) also implies that we can choose $v>0$ large enough so that $-(v+$ $\beta+k)<-L$. Thus, for $v>>0$ we have $\left|s_{2}(-v)\right| \leq \tilde{B}$.

We have proven that $P Q=\sum_{v \in \mathbb{Z}} c_{v} D^{v}$ belongs to $\widehat{\Psi}(\hat{A})$. It remains to show that $\widehat{\mathscr{D}}_{\hat{A}}$ is a subalgebra of $\widehat{\Psi}(\hat{A})$. Indeed, it follows from (2.3) and (3.3) that if $v<0$ then $c_{v}=0$, and so $P, Q \in \widehat{\mathscr{D}}_{\hat{A}}$ imply $P Q \in \widehat{\mathscr{D}}_{\hat{A}}$. Finally, if the coefficients $a$ of $P$ and $b$ of $Q$ are smooth functions depending on $x \in O \subset$ $\mathbb{R}^{p}$ such that the constants $A_{P}, B_{p}, M_{P}, N_{P}$ and $L_{p}$ do not depend on $x$, then the bound (3.4), the above uniform estimates for $s_{1}(v)$ and $s_{2}(v)$, and Theorem 2.1, allow us to check that the coefficients of $P Q$ are continuous functions of $a, b$ which depend smoothly on $x$. 
We are ready to define the Mulase group of infinite order pseudo-differential operators. We use obvious adaptations of notations introduced in Subsection 2.1. A crucial remark is that if $B$ is an arbitrary (diffeological) algebra equipped with a (smooth) derivation, then the set $1+\mathscr{I}_{B}$ is a mutiplicative group, see [8], and also a diffeological group, see [13]. In particular, $\operatorname{Int}\left(\mathscr{O}_{\hat{A}} / \mathscr{P}_{\hat{A}}\right)=$ $1+\mathscr{I}_{\mathscr{O}_{\hat{A}} / \mathscr{P}_{\hat{A}}}$ is a group. For $P=\sum_{v \in \mathbb{Z}} a_{v} D^{v} \in \widehat{\Psi}\left(\mathscr{O}_{\hat{A}}\right)$ we set $\pi(P):=\sum_{v \in \mathbb{Z}} \pi\left(a_{v}\right) D^{v}$.

Definition 3.2. We define the spaces

$$
G\left(\mathscr{O}_{\hat{A}}\right)=\left\{P \in \widehat{\Psi}\left(\mathscr{O}_{\hat{A}}\right): \pi(P) \in \operatorname{Int}\left(\mathscr{O}_{\hat{A}} / \mathscr{P}_{\hat{A}}\right)\right\}
$$

and

$$
G_{+}\left(\mathscr{O}_{\hat{A}}\right)=\left\{P \in \widehat{\mathscr{D}}_{\mathscr{O}_{\hat{A}}}: \pi(P)=1\right\} .
$$

Proposition 3.1. The space $G\left(\mathscr{O}_{\hat{A}}\right)$ is a group: each element $P \in G\left(\mathscr{O}_{\hat{A}}\right)$ has an inverse of the form

$$
P^{-1}=\sum_{n \geq 0}(1-P)^{n}
$$

In addition, the space $G_{+}\left(\mathscr{O}_{\hat{A}}\right)$ is a subgroup of $G\left(\mathscr{O}_{\hat{A}}\right)$.

We remark that if $P, Q \in G\left(\mathscr{O}_{\hat{A}}\right)$, then Equation (3.3) for the coefficients of $P Q$ and continuity of $\pi$ imply that $P Q \in G\left(\mathscr{O}_{\hat{A}}\right)$. Thus, the main difficulty in proving Proposition 3.1 is checking that (3.10) makes sense. We need the following two lemmas.

Lemma 3.2. Consider the sequence $Q_{n}=\sum_{v \in \mathbb{Z}} b_{n, v} D^{v}, n \geq 0$, in $\widehat{\Psi}(\hat{A})$. We assume that it is uniformly bounded at $-\infty$, that is, there exists $C>0$ and $L \in \mathbb{Z}^{+}$such that $\left|b_{n, v}\right|<C$ for all $n \geq 1$ and all $v<-L$, and that there exists a positive real number $J$ such that

$$
\hat{\sigma}\left(b_{n, v}\right) \geq J(v+n) \text { for all } v \geq-n .
$$

Then, for each fixed $v \in \mathbb{Z}$ the series $\sum_{n \geq 0} b_{n, v}$ is a well-defined element of $\hat{A}$ and $\sum_{n=0}^{\infty} Q_{n} \in \widehat{\Psi}(\hat{A})$.

Proof. Condition (3.11) implies that

$$
\left|b_{n, v}\right| \leq e^{J(v+n) \ln (c)}=\frac{1}{e^{J(v+n) r}} \leq \frac{J^{\prime}}{v+n+1} \text { for all } v \geq-n,
$$

in which $\ln (c)=-r$ and $J^{\prime}=1 / J r$. We use Lemma 1. Let us assume first that $v \geq 0$. Hence, (3.12) implies that $\left|b_{n, v}\right| \leq \frac{J^{\prime}}{v+n+1}$ for all $n>0$, and so $\lim _{n \rightarrow \infty} b_{n, v}=0$. This means that $\sum_{n \geq 0} b_{n, v} \in \hat{A}$. Now assume that $v=-\mu<0$. Then, only a finite number of integers $n \in \mathbb{Z}^{+}$will not satisfy $v \geq-n$. It follows that $\lim _{n \rightarrow \infty} b_{n, v}=0$, and again we conclude that $\sum_{n \geq 0} b_{n, v} \in \hat{A}$.

Now we consider the formal sum

$$
\sum_{n=1}^{\infty} Q_{n}=\sum_{n=1}^{\infty} \sum_{v \in \mathbb{Z}} b_{n, v} D^{v}=\sum_{v \in \mathbb{Z}}\left(\sum_{n=1}^{\infty} b_{n, v}\right) D^{v} .
$$

Since $\sum_{n \geq 0} b_{n, v} \in \hat{A}$, Lemma 1 and (3.12) imply that

$$
\left|\sum_{n \geq 0} b_{n, v}\right|<\max _{n \geq 0}\left|b_{n, v}\right| \leq \max _{n \geq 0} \frac{J^{\prime}}{v+n+1} \text { for } v \geq 1 .
$$

Thus, $\left|\sum_{n \geq 0} b_{n, v}\right| \leq \frac{J^{\prime}}{v}$ for all $v>0$, and $\left|\sum_{n \geq 0} b_{n, v}\right|<\max _{n \geq 0}\left|b_{n, v}\right|<C$ for $v<-L$. Thus, $\sum_{n=1}^{\infty} Q_{n} \in \widehat{\Psi}(\hat{A})$. 
Lemma 3.3. Let $R=\sum_{v \in \mathbb{Z}} \tilde{b}_{v} D^{v} \in \widehat{\Psi}\left(\mathscr{O}_{\hat{A}}\right)$ and let $\left\{c_{n}\right\}_{n \geq 0}$ be a sequence of scalars in $K$ such that $c_{0}=1$ and $c_{n} \neq 0$ for $n \geq 1$. If $\hat{\sigma}\left(\tilde{b}_{v}\right) \geq 1$ for all $v \geq 0$, then $\sum_{n \geq 0} c_{n} R^{n} \in G\left(\mathscr{O}_{\hat{A}}\right)$.

Proof. We show that the sequence $Q_{n}=c_{n} R^{n}, n \geq 0$, satisfies the conditions of the previous lemma. Since $R \in \widehat{\Psi}\left(\mathscr{O}_{\hat{A}}\right)$, there exist $A_{R}, B_{R} \in \mathbb{R}^{+}$and $M_{R}, N_{R}, L_{R} \in \mathbb{Z}^{+}$so that $\left|\tilde{b}_{v}\right|<\frac{A_{R}}{v-N_{R}}$ for all $\left.v\right\rangle$ $M_{R}>N_{R}$ and $\left|\tilde{b}_{v}\right|<B_{R}$ for all $v<-L_{R}$. Therefore, there exist $C \in \mathbb{R}^{+}$and $N \in \mathbb{Z}^{+}$such that $\hat{\sigma}\left(\tilde{b}_{v}\right)>C v-N$ for all $v \gg 0$.

We claim that there exists a number $J>0$ such that $\hat{\sigma}\left(\tilde{b}_{v}\right) \geq J(v+1)$ for all $v \geq-1$. The claim is trivial if $v=-1$ since $R \in \widehat{\Psi}\left(\mathscr{O}_{\hat{A}}\right)$ by hypothesis; if $v \geq 0$, we fix $0<\varepsilon<C$. Then, there exists $\widetilde{M}>0$ such that

$$
C-\varepsilon \leq \frac{C v-N}{v+1} \leq C+\varepsilon
$$

for all $v>\widetilde{M}$, and we choose $M \geq \max \left\{\widetilde{M}, M_{R}\right\}$ so that we also have the estimate $\hat{\sigma}\left(\tilde{b}_{v}\right)>C v-N$ for all $v \geq M$. Then, on the one hand, if $0 \leq v \leq M$ we have the inequality $0<\frac{C}{C+C M+N}(v+1)<1$, and therefore (since we are assuming $\hat{\sigma}\left(\tilde{b}_{v}\right) \geq 1$ ) we conclude that $\hat{\sigma}\left(\tilde{b}_{v}\right) \geq \frac{C}{C+C M+N}(v+1)$ for $-1 \leq v \leq M$. On the other hand, if $v>M$, then (3.13) implies that $\hat{\sigma}\left(\tilde{b}_{v}\right) \geq C v-N>(C-\varepsilon)(v+$ 1). Our claim is proved if we choose $J=\min \{C /(C+C M+N), C-\varepsilon\}$.

Now we consider the sequence $Q_{n}=c_{n} R^{n}$ and we write $Q_{n}=\sum_{v \in \mathbb{Z}} b_{n, v} D^{v}$. We show by induction that

$$
\hat{\sigma}\left(b_{n, v}\right) \geq J(v+n) \text { for all } v \geq-n .
$$

If $n=1$, then $\hat{\sigma}\left(b_{1, v}\right)=\hat{\sigma}\left(c_{1} \tilde{b}_{v}\right)=\hat{\sigma}\left(\tilde{b}_{v}\right) \geq J(v+1)$ for all $v \geq-1$, as we just showed.

We assume that (3.14) holds for $n$ and we show that $\hat{\sigma}\left(b_{n+1, v}\right) \geq J(v+n+1)$ for $v \geq-n-1$. We have $Q_{n+1}=c_{n+1} R^{n+1}=\frac{c_{n+1}}{c_{n}} R Q_{n}$, that is,

$$
Q_{n+1}=\frac{c_{n+1}}{c_{n}}\left(\sum_{\alpha \in \mathbb{Z}} \tilde{b}_{\alpha} D^{\alpha}\right)\left(\sum_{\mu \in \mathbb{Z}} b_{n, \mu} D^{\mu}\right)=\frac{c_{n+1}}{c_{n}} \sum_{v \in \mathbb{Z}}\left[\sum_{\alpha \in \mathbb{Z}} \sum_{i=0}^{\infty}\left(\begin{array}{c}
\alpha \\
i
\end{array}\right) \tilde{b}_{\alpha} b_{n, v-\alpha+i}^{(i)}\right] D^{v} .
$$

The coefficient of $D^{v}$ in (3.15) can be written as follows:

$$
\begin{array}{r}
\frac{c_{n}}{c_{n+1}} b_{n+1, v}=\sum_{\alpha=0}^{v+n} \sum_{i=0}^{\alpha}\left(\begin{array}{c}
\alpha \\
i
\end{array}\right) \tilde{b}_{\alpha} b_{n, v-\alpha+i}^{(i)}+\sum_{\alpha=v+n+1}^{\infty} \sum_{i=0}^{\alpha}\left(\begin{array}{c}
\alpha \\
i
\end{array}\right) \tilde{b}_{\alpha} b_{n, v-\alpha+i}^{(i)}+ \\
\sum_{l=1}^{\infty} \sum_{i=0}^{l-1}\left(\begin{array}{c}
i-l \\
i
\end{array}\right) \tilde{b}_{i-l} b_{n, v+l}^{(i)},
\end{array}
$$

where $l=\beta+i$. We bound each one of these three summations: Set

$$
A=\sum_{\alpha=0}^{v+n} \sum_{i=0}^{\alpha}\left(\begin{array}{c}
\alpha \\
i
\end{array}\right) b_{\alpha} b_{n, v-\alpha+i}^{(i)} .
$$

Then, $0 \leq \alpha \leq v+n$ and $i \geq 0$, so that $v-\alpha+i \geq-n$. The induction hypothesis implies that $\hat{\sigma}\left(b_{n, v-\alpha+i}\right) \geq J(v-\alpha+i+n) \geq J(v-\alpha+n)$. Since $D$ and $\hat{\sigma}$ are compatible, we obtain $\hat{\sigma}\left(b_{n, v-\alpha+i}^{(i)}\right) \geq J(v-\alpha+n)$. Moreover, since $\alpha \geq 0$, the $n=1$ induction step implies that $\hat{\sigma}\left(\tilde{b}_{\alpha}\right) \geq$ $J(\alpha+1)$. Therefore, $\hat{\sigma}\left(\tilde{b}_{\alpha} b_{n, v-\alpha+i}^{(i)}\right) \geq \hat{\sigma}\left(\tilde{b}_{\alpha}\right)+\hat{\sigma}\left(b_{n, v-\alpha+i}^{(i)}\right) \geq J(\alpha+1)+J(v-\alpha+n)$, and so $\hat{\sigma}(A) \geq J(v+n+1)$. 
Next, we set

$$
B=\sum_{\alpha=v+n+1}^{\infty} \sum_{i=0}^{\alpha}\left(\begin{array}{c}
\alpha \\
i
\end{array}\right) \tilde{b}_{\alpha} b_{n, v-\alpha+i}^{(i)}
$$

Then,

$$
\hat{\sigma}(B)=\hat{\sigma}\left(\lim _{N \rightarrow \infty} \sum_{\alpha=v+n+1}^{N} \sum_{i=0}^{\alpha}\left(\begin{array}{c}
\alpha \\
i
\end{array}\right) \tilde{b}_{\alpha} b_{n, v-\alpha+i}^{(i)}\right)=\lim _{N \rightarrow \infty} \hat{\sigma}\left(\sum_{\alpha=v+n+1}^{N} \sum_{i=0}^{\alpha}\left(\begin{array}{c}
\alpha \\
i
\end{array}\right) \tilde{b}_{\alpha} b_{n, v-\alpha+i}^{(i)}\right) .
$$

Now,

$$
\begin{aligned}
\hat{\sigma}\left(\sum_{\alpha=v+n+1}^{N} \sum_{i=0}^{\alpha}\left(\begin{array}{c}
\alpha \\
i
\end{array}\right) b_{\alpha} b_{n, v-\alpha+i}^{(i)}\right) & \geq \min _{v+n+1 \leq \alpha \leq N} \min _{0 \leq i \leq \alpha} \hat{\sigma}\left(\tilde{b}_{\alpha} b_{n, v-\alpha+i}^{(i)}\right) \\
& \geq \min _{v+n+1 \leq \alpha \leq N} \min _{0 \leq i \leq \alpha}\left\{\hat{\sigma}\left(\tilde{b}_{\alpha}\right)+\hat{\sigma}\left(b_{n, v-\alpha+i}^{(i)}\right)\right\} \\
& \geq \min _{v+n+1 \leq \alpha \leq N} \min _{0 \leq i \leq \alpha} \hat{\sigma}\left(\tilde{b}_{\alpha}\right),
\end{aligned}
$$

since $R$ (and hence $R^{n}$ ) belongs to $G\left(\mathscr{O}_{\hat{A}}\right)$ and therefore $\hat{\sigma}\left(b_{n, v-\alpha+i}^{(i)}\right) \geq 0$. Using the $n=1$ induction step (which we can, because $v+n+1 \geq 0$ ) we obtain

$$
\min _{v+n+1 \leq \alpha \leq N} \min _{0 \leq i \leq \alpha} \hat{\sigma}\left(\tilde{b}_{\alpha}\right)=\min _{v+n+1 \leq \alpha \leq N} \hat{\sigma}\left(\tilde{b}_{\alpha}\right) \geq \min _{v+n+1 \leq \alpha \leq N} J(\alpha+1) \geq J(v+n+2),
$$

and therefore $\hat{\sigma}(B) \geq J(v+n+2) \geq J(v+n+1)$.

Finally, we set

$$
C=\sum_{l=1}^{\infty} \sum_{i=0}^{l-1}\left(\begin{array}{c}
i-l \\
i
\end{array}\right) b_{i-l} b_{n, v+l}^{(i)}
$$

Then, using again the fact that we are considering formal pseudo-differential operators with nonnegative valuations, we have,

$$
\begin{aligned}
\hat{\sigma}\left(\sum_{l=1}^{N} \sum_{i=0}^{l-1}\left(\begin{array}{c}
i-l \\
i
\end{array}\right) b_{i-l} b_{n, v+l}^{(i)}\right) & \geq \min _{1 \leq l \leq N} \min _{0 \leq i \leq l-1}\left\{\hat{\boldsymbol{\sigma}}\left(\tilde{b}_{i-l}\right)+\hat{\sigma}\left(b_{n, v+l}^{(i)}\right)\right\} \\
& \geq \min _{1 \leq l \leq N} \min _{0 \leq i \leq l-1} \hat{\boldsymbol{\sigma}}\left(b_{n, v+l}^{(i)}\right) .
\end{aligned}
$$

Since $l \geq 1$ and we are assuming $v \geq-n-1$, we have $v+l \geq v+1 \geq-n$. The induction hypothesis then implies that $\hat{\sigma}\left(b_{n, v+l}^{(i)}\right) \geq J(v+l+n) \geq J(v+n+1)$, and we conclude that $\hat{\sigma}(C) \geq J(v+n+1)$.

Collecting these results we have

$$
\hat{\sigma}\left(b_{n+1, v}\right)=\hat{\sigma}\left(\left(c_{n} / c_{n+1}\right) b_{n+1, v}\right)=\hat{\sigma}(A+B+C) \geq \min \{\hat{\sigma}(A), \hat{\sigma}(B), \hat{\sigma}(C)\} \geq J(v+n+1),
$$

and we have proven (3.14). 
Since we easily check that $\left|b_{n, v}\right| \leq 1$ for all $n \geq 1$ and $v \in \mathbb{Z}$, Lemma 3 implies that $\sum_{n=0}^{\infty} Q_{n} \in$ $\widehat{\Psi}(\hat{A})$. It remains to show that $\sum_{n=0}^{\infty} Q_{n} \in G\left(\mathscr{O}_{\hat{A}}\right)$. We have,

$$
\sum_{n=0}^{\infty} Q_{n}=1+\sum_{n=1}^{\infty} Q_{n}=1+\sum_{v \in \mathbb{Z}}\left(\sum_{n=1}^{\infty} b_{n, v}\right) D^{v},
$$

and

$$
\hat{\sigma}\left(\sum_{n=1}^{\infty} b_{n, v}\right)=\lim _{N \rightarrow \infty} \hat{\sigma}\left(\sum_{n=1}^{N} b_{n, v}\right) \geq \lim _{N \rightarrow \infty} \min _{1 \leq n \leq N} \hat{\sigma}\left(b_{n, v}\right) .
$$

This limit is non-negative because we already know that $\left|b_{n, v}\right| \leq 1$ for all $n, v$. Thus, $\sum_{n=0}^{\infty} Q_{n} \in$ $\widehat{\Psi}\left(\mathscr{O}_{\hat{A}}\right)$. Now we consider $v \geq 0$. The estimate (3.14) implies that $\hat{\sigma}\left(b_{n, v}\right) \geq J(n+v)>0$ for all $n \geq 1$, and therefore we conclude, using (3.17), that $\hat{\sigma}\left(\sum_{n=1}^{\infty} b_{n, v}\right) \geq J(v+1)$. Now, (3.16) tells us that $\pi\left(\sum_{n=0}^{\infty} Q_{n}\right)=1+\sum_{v \in \mathbb{Z}} \pi\left(\sum_{n=1}^{\infty} b_{n, v}\right) D^{v}$ (as $\pi(1)=1$ ), and so $\sum_{n=0}^{\infty} Q_{n}$ belongs to $G\left(\mathscr{O}_{\hat{A}}\right)$.

Proof of Proposition 3.1: We consider $P=\sum_{v \in \mathbb{Z}} a_{v} D^{v} \in G\left(\mathscr{O}_{\hat{A}}\right)$. The formal inverse of $P$ is given by the simple calculation

$$
1=\sum_{n=0}^{\infty}(1-P)^{n}-\sum_{n=1}^{\infty}(1-P)^{n}=\sum_{n=0}^{\infty}(1-P)^{n}-(1-P) \sum_{n=0}^{\infty}(1-P)^{n}=P \sum_{n=0}^{\infty}(1-P)^{n} .
$$

We show that the series $\sum_{n=0}^{\infty}(1-P)^{n}$ belongs to $G\left(\mathscr{O}_{\hat{A}}\right)$ : Set $Q=1-P=\sum_{v \in \mathbb{Z}} b_{v} D^{v} \in \widehat{\Psi}\left(\mathscr{O}_{\hat{A}}\right)$. As $\pi(Q)=1-\pi(P) \in \mathscr{I}_{\mathscr{O}_{\hat{A}} / \mathscr{P}_{\hat{A}}}$, and the range of $\hat{\sigma}$ is $\mathbb{Z}$, we have $\hat{\sigma}\left(b_{v}\right) \geq 1$ for $v \geq 0$, and so the claim follows from Lemma 3.3.

Now we note that if $P \in G_{+}\left(\mathscr{O}_{\hat{A}}\right)$ then $\sum_{n>0}(1-P)^{n} \in G\left(\mathscr{O}_{\hat{A}}\right)$, simply because $G_{+}\left(\mathscr{O}_{\hat{A}}\right)$ is contained in $G\left(\mathscr{O}_{\hat{A}}\right)$. Also, $\pi(1-P)=0$, so that continuity of $\pi$ yields $\pi\left(\left(\sum_{n \geq 0}(1-P)^{n}\right)\right)=1$ and we conclude that $P^{-1} \in G_{+}\left(\mathscr{O}_{\hat{A}}\right)$. Finally, let $P_{1}=\sum_{\alpha \geq 0} a_{\alpha} D^{\alpha}$ and $P_{2}=\sum_{\beta \geq 0} b_{\beta} D^{\beta}$ in $G_{+}\left(\mathscr{O}_{\hat{A}}\right)$. Then, $P_{1} P_{2}=\sum_{v \geq 0} c_{v} D^{v}$ with

$$
c_{v}=\sum_{\alpha=0}^{\infty} \sum_{k=0}^{\alpha}\left(\begin{array}{l}
\alpha \\
k
\end{array}\right) a_{\alpha} b_{v-\alpha+k}^{(k)}
$$

and we conclude from the proof of Lemma 2 that $\hat{\sigma}\left(c_{v}\right) \geq \min _{\alpha \geq 0} \min _{0 \leq k \leq \alpha}\left\{\hat{\sigma}\left(a_{\alpha}\right)+\right.$ $\left.\hat{\sigma}\left(b_{v-\alpha+k}\right)\right\}$. We bound $S=\hat{\sigma}\left(a_{\alpha}\right)+\hat{\sigma}\left(b_{v-\alpha+k}^{(k)}\right)$. If $\alpha \neq 0$, then the definition of $G_{+}\left(\mathscr{O}_{\hat{A}}\right)$ implies that $\sigma\left(a_{\alpha}\right) \geq 1$, and so $S \geq 1$ for all $k \geq 0$. On the other hand, if $\alpha=0$, then $\hat{\sigma}\left(a_{\alpha}\right)=0$ and $S=\hat{\sigma}\left(b_{v}\right)$. If $v>0$ we have, again because of the definition of $G_{+}\left(\mathscr{O}_{\hat{A}}\right)$, that $S \geq \hat{\sigma}\left(b_{v}\right) \geq 1$ and therefore $\pi\left(P_{1} P_{2}\right)=\pi\left(c_{0}\right)$. Formula (3.18) for $v=0$ and the above computations imply that $\pi\left(c_{0}\right)=\pi\left(a_{0} b_{0}\right)=\pi\left(a_{0}\right) \pi\left(b_{0}\right)=1$, and so $P_{1} P_{2} \in G_{+}\left(\mathscr{O}_{\hat{A}}\right)$.

Proposition 3.2. The group $G_{-}\left(\mathscr{O}_{\hat{A}}\right)=1+\mathscr{I}_{\mathscr{O}_{\hat{A}}}$ is a formal Lie group with Lie algebra $\mathscr{I}_{\mathscr{O}_{\hat{A}}}$. The exponential map

$$
\exp : P \in \mathscr{I}_{\mathscr{O}_{\hat{A}}} \mapsto \sum_{n \in \mathbb{N}} \frac{(s P)^{n}}{n !} \in G_{-}\left(\mathscr{O}_{\hat{A}}\right)
$$

in which $s \in K$, is one-to-one and onto with inverse given by the clasical logarithmic series log, and both $\exp$ and $\log$ are smooth. As a consequence, inversion is smooth in $G_{-}\left(\mathscr{O}_{\hat{A}}\right)$. 
Proof. (Sketch) Once the rest of the proposition is checked, smoothness of exp and log follows because of the choice of diffeology on $\hat{\Psi}(\hat{A})$; this diffeology is defined coefficientwise from the diffeology of $\hat{A}$ via product and pull-back (or initial) diffeologies, see [10] and also [13] for related constructions.

It follows from our remarks above Definition 3.2 that the space $1+\mathscr{I}_{\mathscr{O}_{\hat{A}}}$ is a multiplicative (diffeological) group. We consider a curve $g(s)$ in $1+\mathscr{I}_{\mathscr{O}_{\hat{A}}}$ with $g(0)=1$. Then, it is obvious that $g^{\prime}(0) \in \mathscr{I}_{\mathscr{O}_{\hat{A}}}$. On the other hand, if $P=a_{1} D^{-1}+a_{2} D^{-2}+\cdots \in \mathscr{I}_{\mathscr{A}_{\hat{A}}}$, then Lemma 3.3 implies that $\exp (s P)=\sum_{n \geq 0}\left(s^{n} / n !\right) P^{n}, s \in K$, is in $G\left(\mathscr{O}_{\hat{A}}\right)$. Since only non-positive powers of $D$ appear in $P$, we conclude that $\exp (s P) \in 1+\mathscr{I}_{\mathscr{O}_{\hat{A}}}$, and moreover the algebraic derivative (i.e., the operator $d / d s$ acting on formal power series in the variable $s$ ) of the curve $s \mapsto \exp (s P)$ at $s=0$ is $P$.

The following result is our version of Mulase's factorization theorem in the context of diffeological topological algebras.

Theorem 3.1. For any $U \in G\left(\mathscr{O}_{\hat{A}}\right)$ there exist unique $W \in G_{-}\left(\mathscr{O}_{\hat{A}}\right)$ and $Y \in G_{+}\left(\mathscr{O}_{\hat{A}}\right)$ such that

$$
U=W^{-1} Y
$$

In other words, there exists a unique global factorization of the formal Lie group $G\left(\mathscr{O}_{\hat{A}}\right)$ as a product group, $G\left(\mathscr{O}_{\hat{A}}\right)=G_{-}\left(\mathscr{O}_{\hat{A}}\right) G_{+}\left(\mathscr{O}_{\hat{A}}\right)$. Moreover, the map $U \mapsto(W, Y)$ are smooth with respect to the diffeologies inherited from the diffeology of $\hat{\Psi}(\hat{A})$.

Proof. Let us assume first that $W_{1}^{-1} Y_{1}=W_{2}^{-1} Y_{2}$; then, $W_{1} W_{2}^{-1}=Y_{1} Y_{2}^{-1}$. Since $W_{1} W_{2}^{-1} \in G_{-}\left(\mathscr{O}_{\hat{A}}\right)$ and $W_{1} W_{2}^{-1} \in G_{+}\left(\mathscr{O}_{\hat{A}}\right)$, we must have $W_{1} W_{2}^{-1}=Y_{1} Y_{2}^{-1}=1$, and therefore $W_{1}=W_{2}$ and $Y_{1}=Y_{2}$. This shows uniqueness.

In order to prove the existence of the decomposition (3.19), we solve the equation $W U=Y$ for an unknown $W \in G_{-}\left(\mathscr{O}_{\hat{A}}\right)$. Let $U=\sum_{\beta \in \mathbb{Z}} u_{\beta} D^{\beta} \in G\left(\mathscr{O}_{\hat{A}}\right)$ and $W=1+\sum_{\alpha=1}^{\infty} w_{\alpha} D^{-\alpha}$. We write

$$
W U=\sum_{\beta \in \mathbb{Z}} u_{\beta} D^{\beta}+\sum_{\gamma \in \mathbb{Z}}\left(\sum_{\alpha=1}^{\infty} \sum_{i=0}^{\infty}\left(\begin{array}{c}
-\alpha \\
i
\end{array}\right) w_{\alpha} u_{\gamma+\alpha+i}^{(i)}\right) D^{\gamma} .
$$

We know that $W U \in \widehat{\Psi}(\hat{A})$; we need to show that $W U \in G_{+}\left(\mathscr{O}_{\hat{A}}\right)$ so that, in particular, $W U$ should not have negative powers of $D$. Hence, the formal pseudo-differential operator $W$ should solve the linear system of equations

$$
u_{-\beta}+\sum_{\alpha=1}^{\infty} \sum_{i=0}^{\infty}\left(\begin{array}{c}
-\alpha \\
i
\end{array}\right) w_{\alpha} u_{\alpha-\beta+i}^{(i)}=0
$$

for $\beta=1,2,3, \cdots$, in which the unknowns are the $w_{\alpha}$ 's belonging to $\mathscr{O}_{\hat{A}}$. Following Mulase, see [15], we define $\vec{u}=\left(u_{-1}, u_{-2}, u_{-3}, \ldots\right), \vec{w}=\left(w_{1}, w_{2}, w_{3}, \ldots\right)$, and

$$
M=\left[\sum_{i=0}^{\infty}\left(\begin{array}{c}
-\alpha \\
i
\end{array}\right) u_{\alpha-\beta+i}^{(i)}\right]_{\alpha, \beta=1,2,3, \cdots} .
$$

The coefficients of $\vec{u}$ and $M$ are smooth with respect to the coefficients of $U$, and hence $\vec{u}$ and $M$ can be understood as objects in the spaces of sequences $\hat{A}^{-\mathbb{N}^{*}}$ and $\hat{A}^{\mathbb{Z}^{2}}$ which are coefficientwise smooth for the infinite product (uniform) diffeologies. Equation (3.20) becomes $\vec{w} M=-\vec{u}$, and its solution should be $\vec{w}=-\vec{u} M^{-1}$, as long as $M$ is invertible. We now prove that $M^{-1}=\sum_{n=0}^{\infty}(I d-$ 
$M)^{n}$, where $I d$ is the infinite identity matrix. We define $N=I d-M=\left[a_{\mu v}\right]_{\mu, v=1,2,3, \ldots}$ and $N^{n}=$ $\left[a_{n, \mu v}\right]_{\mu, v=1,2,3, \ldots}$. Thus,

$$
a_{\mu v}=\delta_{\mu v}-\sum_{i=0}^{\infty}\left(\begin{array}{c}
-\mu \\
i
\end{array}\right) u_{\mu-v+i}^{(i)}
$$

We note that $\hat{\sigma}\left(a_{\mu v}\right) \geq 0$, and also $\hat{\sigma}\left(a_{n, \mu v}\right) \geq 0$ for all $n \geq 1$. We can be more precise if we examine projections. First, note that

$$
\pi\left(a_{\mu \mu}\right)=\pi\left(1-\sum_{i=0}^{\infty}\left(\begin{array}{c}
-\mu \\
i
\end{array}\right) u_{i}^{(i)}\right)=\pi\left(-\sum_{i=1}^{\infty}\left(\begin{array}{c}
-\mu \\
i
\end{array}\right) u_{i}^{(i)}\right)=0,
$$

since $U=\sum_{\beta \in \mathbb{Z}} u_{\beta} D^{\beta} \in G\left(\mathscr{O}_{\hat{A}}\right)$ implies $\pi(U)=1+\pi\left(u_{-1}\right) D^{-1}+\pi\left(u_{-2}\right) D^{-2}+\cdots$, so that $\pi\left(u_{i}\right)=$ 0 for $i>0$ and - using $\hat{\sigma}\left(u_{i}^{(i)}\right) \geq \sigma\left(u_{i}\right)$ - we have $\pi\left(u_{i}^{(i)}\right)=0$ as well. Also, if $\mu>v$, then

$$
\pi\left(a_{\mu \nu}\right)=\pi\left(-\sum_{i=0}^{\infty}\left(\begin{array}{c}
-\mu \\
i
\end{array}\right) u_{\mu-v+i}^{(i)}\right)=0
$$

since in this case $\mu-v+i \geq 1$ and so $\pi\left(u_{\mu-v+i}^{(i)}\right)=0$ for all $i$ by the same argument as above. In other words, we have proven that $\hat{\sigma}\left(a_{\mu v}\right) \geq 1$ for $\mu \geq v$.

Now we make two claims. First, there exists $J \in \mathbb{R}^{+}$such that $\hat{\sigma}\left(a_{\mu v}\right) \geq J(\mu-v+1)$ for all $\mu-v \geq-1$ :

Indeed, if $v-\mu=-1$, then any $J$ satisfies our claim. If $v=\mu$ we already saw that $\hat{\sigma}\left(a_{\mu \mu}\right) \geq$ $1=1(\mu-\mu+1)$. If $\mu>v$, we have, using the proof of Lemma 3.3 and the fact that $\hat{\sigma}\left(u_{i}\right) \geq 1$ for all $i \geq 0$

$$
\hat{\sigma}\left(u_{\mu-v+i}^{(i)}\right) \geq \hat{\sigma}\left(u_{\mu-v+i}\right) \geq \tilde{J}(\mu-v+i+1) \geq \tilde{J}(\mu-v+1)
$$

for some $\tilde{J}>0$, since $\mu-v+i \geq-1$. Therefore $\hat{\sigma}\left(u_{\mu-v+i}^{(i)}\right) \geq \tilde{J}(\mu-v+1)$ for all $i$, and it follows that $\hat{\sigma}\left(a_{\mu v}\right) \geq \tilde{J}(\mu-v+1)$. We choose $J=\min \{\tilde{J}, 1\}$.

Second, we claim that for every $n \geq 1$, we have $\hat{\sigma}\left(a_{n, \mu v}\right) \geq J(\mu-v+n)$ if $\mu-v \geq-n$ :

We use induction. If $n=1$, then $a_{1, \mu v}=a_{\mu v}$ and the claim reduces to what we just proved. We assume that $\hat{\sigma}\left(a_{n, \mu v}\right) \geq J(\mu-v+n)$ whenever $\mu-v \geq-n, n \geq 1$. Since $N^{n+1}=N^{n} N$ we have

$$
a_{n+1, \mu v}=\sum_{l=1}^{\infty} a_{n, \mu l} a_{l v}=\sum_{l=1}^{v-1} a_{n, \mu l} a_{l v}+\sum_{l=v}^{\mu+n} a_{n, \mu l} a_{l v}+\sum_{l=\mu+n+1}^{\infty} a_{n, \mu l} a_{l v}
$$

We can assume that $\mu-v \geq-n-1$, since the case $\mu-v=-n$ is trivial by hypothesis. We need to estimate each of the three sums above. These estimations are carried out in Mulase's paper [15, p. 27] for a specific valuation, but his arguments are valid in general.

Next, we show that $b_{\mu v}=\sum_{n=0}^{\infty} a_{n, \mu v}$ belongs to $\mathscr{O}_{\hat{A}}$ for $\mu, v \geq 1$. If $\mu-v \geq 0$, then $\hat{\sigma}\left(a_{n, \mu v}\right) \geq J(\mu-v+n)$ for all $n \geq 0$. Then, $\lim _{n \rightarrow \infty} \hat{\sigma}\left(a_{n, \mu v}\right)=\infty$ and so $\sum_{n=0}^{\infty} a_{n, \mu v}$ exists in $\hat{A}$. If $\mu-v<0$, then $\hat{\sigma}\left(a_{n, \mu v}\right) \geq J(\mu-v+n)$ for all, but a finite number, of $n$ 's. Again, it follows that $\lim _{n \rightarrow \infty} \hat{\sigma}\left(a_{n, \mu v}\right)=\infty$ and so $b_{\mu v} \in \hat{A}$. Moreover, $\left|b_{\mu v}\right|<\max _{n \geq 0}\left|a_{n, \mu v}\right| \leq 1$, and so $b_{\mu v} \in \mathscr{O}_{\hat{A}}$. 
Thus, we conclude that

$$
\sum_{n=0}^{\infty}(1-M)^{n}=\sum_{n=0}^{\infty} N^{n}=\left[\sum_{n=0}^{\infty} a_{n, \mu v}\right]_{\mu, v \geq 1}
$$

is well defined. As in Prop. 3.1, a trivial computation shows that this matrix is the inverse of $M$.

Now we consider (3.20) or the equivalent equation $\vec{w} M=-\vec{u}$. Then we have

$$
w_{v}=-\sum_{\mu=1}^{\infty} u_{-\mu} b_{\mu v}=-\sum_{\mu=1}^{v} u_{-\mu} b_{\mu v}-\sum_{\mu=v+1}^{\infty} u_{-\mu} b_{\mu v} .
$$

Therefore $w_{v}$ is well defined if the expression $\sum_{\mu=v+1}^{\infty} u_{-\mu} b_{\mu v}$ makes sense. This is clear because $\hat{\sigma}\left(u_{-\mu} b_{\mu v}\right)=\hat{\sigma}\left(u_{-\mu}\right)+\hat{\sigma}\left(b_{\mu v}\right) \rightarrow \infty$ as $\mu \rightarrow \infty$ since $\hat{\sigma}\left(\sum a_{n, \mu v}\right) \geq \min \hat{\sigma}\left(a_{n, \mu v}\right) \geq J(\mu-v+n)$, as $\mu-v>0 \geq-n$. We conclude that $w_{v}$ is a well defined element of $\hat{A}$ (in fact, it belongs to $\mathscr{O}_{\hat{A}}$ ) and so $W=1+\sum_{v=1}^{\infty} w_{v} D^{-v}$ is in $G_{-}\left(\mathscr{O}_{\hat{A}}\right)$.

Now we set $Y=W U$. We note that $W \in G_{-}\left(\mathscr{O}_{\hat{A}}\right) \subseteq \widehat{\Psi}\left(\mathscr{O}_{\hat{A}}\right)$ and that $U \in G\left(\mathscr{O}_{\hat{A}}\right) \subseteq \widehat{\Psi}\left(\mathscr{O}_{\hat{A}}\right)$. Then $W U \in \widehat{\Psi}\left(\mathscr{O}_{\hat{A}}\right)$, and this product does not have negative powers of $D$. So, $Y=W U \in \widehat{\mathscr{D}}_{\hat{A}}$.

We check that $Y$ is in $G_{+}\left(\mathscr{O}_{\hat{A}}\right)$. The calculations that we used to obtain Equation (3.20) and the fact that $\pi(U) \in \operatorname{Int}\left(\mathscr{O}_{\hat{A}} / \mathscr{P}_{\hat{A}}\right)$ imply that

$$
\pi((W U))=1+\pi\left(\sum_{\gamma \geq 0}\left(\sum_{\alpha=1}^{\infty} \sum_{i=0}^{\infty}\left(\begin{array}{c}
-\alpha \\
i
\end{array}\right) w_{\alpha} u_{\gamma+\alpha+i}^{(i)}\right) D^{\gamma}\right) .
$$

We take $\alpha \geq 1$ and $i, \gamma \geq 0$. Then, $\hat{\sigma}\left(w_{\alpha} u_{\gamma+\alpha+i}^{(i)}\right) \geq \hat{\sigma}\left(w_{\alpha}\right)+\hat{\sigma}\left(u_{\gamma+\alpha+i}^{(i)}\right) \geq \hat{\sigma}\left(w_{\alpha}\right)+\hat{\sigma}\left(u_{\gamma+\alpha+i}\right) \geq 1$ since $\gamma+\alpha+i \geq 1$. Therefore, the valuation of the second summation above is greater or equal to one and we conclude that $\pi(W U)=1$. This means that $Y=W U \in G_{+}\left(\mathscr{O}_{\hat{A}}\right)$, as claimed. Finally, we observe that analyzing the algebraic calculations all along the proof, we see that they are all smooth, which proves that the decomposition $U \mapsto(W, Y)$ is smooth.

\section{The initial value problem of the KP hierarchy}

In this final section we propose the following solution to the Cauchy problem for the KP hierarchy.

Theorem 4.1. Consider the KP system of equations

$$
\frac{d L}{d t_{k}}=\left[\left(L^{k}\right)_{+}, L\right]
$$

with initial condition $L(0):=L_{0}=\sum_{v \in \mathbb{Z}} a_{v} D^{v} \in \Psi\left(\mathscr{O}_{\hat{A}}\right)$ such that $\hat{\sigma}\left(a_{v}\right) \geq 1$ for all $v \geq 0$, and let $Y \in G_{+}\left(\mathscr{O}_{\hat{A}}\right)$ and $S \in G_{-}\left(\mathscr{O}_{\hat{A}}\right)$ be the unique solution to the factorization problem

$$
U=\exp \left(t_{k} L_{0}^{k}\right)=\sum_{n \geq 0} \frac{t_{k}^{n}}{n !}\left(L_{0}^{k}\right)^{n}=S^{-1} Y .
$$

The unique solution to Equation (4.1) with $L(0)=L_{0}$ is

$$
L\left(t_{k}\right)=Y L_{0} Y^{-1} \text {. }
$$

Moreover, the map $L_{0} \in \Psi\left(\mathscr{O}_{\hat{A}}\right) \mapsto L \in \widehat{\Psi}\left(\mathscr{O}_{\hat{A}}\right)$ is smooth at each fixed time $t_{k} \in K$. 


\section{Remark 4.1.}

- We have fixed time because the field $K$ is assumed discrete. We could have stated that the solution is smooth in time for the discrete diffeology, but we find this statement of limited interest. On the other hand, if $A$ as an algebra of series as in $[14,15]$, see Remark 2.1, we can work within the framework of Frölicher spaces and then we obtain smoothness with respect to initial condition and time. Full details appear in [12,13].

- The derivation $d / d t_{k}$ is introduced formally via $d / d t_{k}\left(t_{k}^{m}\right)=m t_{k}^{m-1}$, and it is assumed to commute with $D$. More satisfactorily, we could take advantage of the existence of a Hamiltonian formulation for the KP hierarchy, see [8], so that Equation (4.1) becomes an equation for integral curves of an appropriate vector field. We believe that this point of view can be elaborated along the lines of $[9,13]$, but full details remain to be worked out.

- Lemma 3.3 implies that $\exp \left(t_{k} L_{0}^{k}\right)$ exists in $G\left(\mathscr{O}_{\hat{A}}\right)$. Now, in the classical KP hierarchy, Equations (4.1) are gathered into an infinite system, and the solution $L$ depends on a countable family of variables $t_{1}, t_{2}, \cdots$. Following [13], we can easily modify Theorem 4.1 and obtain a solution $L\left(t_{1}, t_{2}, \cdots\right)$. It is enough to consider the operator

$$
U_{\infty}=\exp \left(\sum_{k \in \mathbb{N}} t_{k} L_{0}^{k}\right)
$$

with $t_{0}=1$. This operator is well defined because of Lemma 3.3.

Proof. The proof is modelled after [18], see also [16, Chp. 1]. We set $U=\exp \left(t_{k} L_{0}^{k}\right)$ and we consider $S$ and $Y$ as in the hypotheses of the theorem. We define $L=Y L_{0} Y^{-1}$. Then,

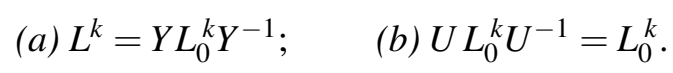

It follows that $L^{k}=Y L_{0}^{k} Y^{-1}=S S^{-1} Y L_{0}^{k} Y^{-1} S S^{-1}=S L_{0}^{k} S^{-1}$. We take $t_{k}$-derivative of $U$ and we obtain $L_{0}^{k} U=-S^{-1} S_{t_{k}} S^{-1} Y+S^{-1} Y_{t_{k}}$. Thus, using $U=S^{-1} Y$, we find the decomposition

$$
S L_{0}^{k} S^{-1}=-S_{t_{k}} S^{-1}+Y_{t_{k}} Y^{-1} \text {. }
$$

Since $S_{t_{k}} S^{-1} \in \mathscr{I}_{\mathscr{O}_{\hat{A}}}$ and $Y_{t_{k}} Y^{-1} \in \mathscr{D}_{\mathscr{O}_{\hat{A}}}=\left\{P \in \Psi\left(\mathscr{O}_{\hat{A}}\right): P\right.$ is a differential operator $\}$, we conclude that $\left(L^{k}\right)_{+}=Y_{t_{k}} Y^{-1}$ and $\left(L^{k}\right)_{-}=-S_{t_{k}} S^{-1}$. Now we take $t_{k}$-derivative of $L$ :

$$
\begin{aligned}
\frac{d L}{d t_{k}} & =Y_{t_{k}} L_{0} Y^{-1}-Y L_{0} Y^{-1} Y_{t_{k}} Y^{-1} \\
& =Y_{t_{k}} Y^{-1} Y L_{0} Y^{-1}-Y L_{0} Y^{-1} Y_{t_{k}} Y^{-1} \\
& =\left(L^{k}\right)_{+} L-L\left(L^{k}\right)_{+} \\
& =\left[\left(L^{k}\right)_{+}, L\right] .
\end{aligned}
$$

We check the initial condition: We have $L(0)=Y(0) L_{0} Y(0)^{-1}$, but $Y(0)=1$ due to the uniqueness of the Mulase factorization.

Finally, since the maps $L_{0} \mapsto U$ and $U \mapsto S$ are smooth, we conclude that the map $L_{0} \mapsto S L_{0} S^{-1}=$ $L$ is smooth.

\section{Acknowledgements}

A.ER has been supported by the Proyecto Basal USA 1555; J.-P.M. and E.G.R. have been partially supported by CONICYT (Chile) via the FONDECYT operating grant \# 1161691. 


\section{References}

[1] J.E. Andersen, G. Borot, L.O. Chekhov, and N. Orantin, The ABCD of topological recursion, ArXiv: 1703.03307.

[2] C. Blohmann, M.C. Barbosa Fernandes and A. Weinstein, Groupoid symmetry and constraints in General Relativity, Commun. Contemp. Math. 15, 1250061 (2013) [25 pages].

[3] S. Bosch, U. Güntzer and R. Remmert, Non-Archimedean Analysis, Springer-Verlag, 1984.

[4] K.T. Chen, Iterated Path Integrals, Bulletin AMS 83 (1977), 831-879.

[5] F.A. Berezin and A.M. Perelomov, Group-theoretical interpretation of the Korteweg-de Vries type equations, Comm. Math. Phys. 74 (1980), 12-140.

[6] E.E. Demidov, On the Kadomtsev-Petviashvili hierarchy with a noncommutative timespace, Funct. Anal. Appl. 29 (1995), 131-133.

[7] E.E. Demidov, Noncommutative deformation of the Kadomtsev-Petviashvili hierarchy, J. Math. Sci. (New York) 88 (1998), 520-536 (English).

[8] L.A. Dickey, Soliton equations and Hamiltonian systems, second edition, World Scientific, 2003.

[9] A. Eslami Rad and E. G. Reyes, The Kadomtsev-Petviashvili hierarchy and the Mulase factorization of formal Lie groups, Geometric Mechanics 5 (2013), 345-364.

[10] P. Iglesias-Zemmour, Diffeology, AMS Mathematical Monographs 185 (2013).

[11] B.A. Khesin and R. Wendt, The geometry of infinite-dimensional groups, Springer-Verlag, 2009.

[12] J.-P. Magnot, Ambrose-Singer theorem on diffeological bundles and complete integrability of KP equations, Int. J. Geom. Meth. Mod. Phys. 10, no 9 (2013) Article ID 1350043.

[13] J.-P. Magnot and E.G. Reyes, Well-posedness of the Kadomtsev-Petviashvili hierarchy, Mulase factorization, and Frölicher Lie groups, arXiv: 1608.03994.

[14] M. Mulase, Complete integrability of the Kadomtsev-Petvishvili equation, Advances in Math. 54 (1984), 57-66.

[15] M. Mulase, Solvability of the super KP equation and a generalization of the Birkhoff decomposition, Invent. Math. 92 (1988), 1-46.

[16] A.M. Perelomov, Integrable systems of classical mechanics and Lie algebras, Birkhäuser Verlag, 1990.

[17] A. Pressley and G.B. Segal, Loop groups, Oxford University Press, 1986.

[18] A.G. Reyman and M.A. Semenov-Tian-Shansky, Reduction of Hamiltonian Systems, Affine Lie Algebras and Lax Equations II, Invent. Math. 63 (1981), 423-432.

[19] M. Sakakibara, Factorization methods for noncommutative KP and Toda hierarchy, J. Phys. A: Math. Gen. 37 (2004), L599-L604.

[20] W.H. Schikhof, Ultrametric calculus. An introduction to p-adic analysis, Cambridge, 1984.

[21] J.-M. Souriau, Un algorithme générateur de structures quantiques; Astérisque, Hors Série, 341-399 (1985).

[22] I.A.B. Strachan and D. Zuo, Integrability of the Frobenius algebra-valued Kadomtsev-Petviashvili hierarchy, J. Math. Phys. 56 (2015), 113509.

[23] K. Takasaki, A New Approach to the Self-Dual Yang-Mills Equations, Commun. Math. Phys. 94 (1984), $35-59$.

[24] K. Takasaki, Nonabelian KP hierarchy with Moyal algebraic coefficients, J. Geom. Phys. 14 (1994), 332-364.

[25] D. Tanré, Homotopie Rationnelle: Modèles de Chen, Quillen, Sullivan, LNM 1025, Springer-Verlag, 1983.

[26] J. Watts, Diffeologies, differentiable spaces and symplectic geometry, University of Toronto, $\mathrm{PhD}$ thesis, arXiv:1208.3634v1.

[27] H. Zhang, Hamiltonian Structures and Integrability of Frobenius Algebra-Valued $(n, m)^{\text {th }} \mathrm{KdV}$ Hierarchy, J. Nonlinear Math. Phys. 24 (2017) 315-327. 